\title{
COMPARATIVE STUDY OF SPAN 40 AND SPAN 60 BASED SOY-GELS FOR TOPICAL DRUG DELIVERY
}

\author{
SHUBHAM MUKHERJEE*, DIPANJANA ASH, SUTAPA BISWAS MAJEE, GOPA ROY BISWAS \\ Department of Pharmacy, NSHM Knowledge Campus, Kolkata, Group of Institutions, Kolkata, West Bengal, India. \\ Email: rx.shubhamm@gmail.com
}

Received: 11 April 2019, Revised and Accepted: 01 May 2019

\begin{abstract}
Objective: Hydrogels or emulgels are recommended for topical application to elicit a local effect. However, they suffer from stability problems. The present study deals with the formulation and comparison of thermally stable soybean oil-based novel topical organogels (soy-gels) using two different gelators (Span 40 and Span 60) for controlled drug delivery.

Methods: Soy-gels (8 batches) were developed with Span 40 and Span 60 by solid fiber mechanism and characterized for viscosity, gelation kinetics behavior, gel-sol transition parameters, drug content, in vitro drug release pattern, and changes occurring during accelerated thermal stability studies.

Results: Fourier transformed infrared spectroscopic confirmed the compatibility among the organogel components and paracetamol. The formulations exhibited skin and hemocompatibility. The viscosity of Span 60 based soy-gels was found to be approximately 10 times higher than those of Span 40 based formulations. In comparison to Span 40, Span 60 induced faster gelation (3-6 min) of soybean oil at lower concentration (16\% w/v) forming less flexible but thermally more stable soy-gels demonstrating higher $\mathrm{T}_{\mathrm{g}}$ values. Higher flexibility and lower viscosity accounted for improved drug diffusion (both Fickian and non-Fickian) from Span 40 gels of varying concentrations in pH 5.8. However, zero-order drug release was observed in organogel with $18 \% \mathrm{w} / \mathrm{v}$ Span 40 only and all Span 60-based formulations except the one with $22 \%$ w/v Span 60 . Non-Fickian drug diffusion occurred from Span 60 based soy-gels. A gradual increase in gelation time was observed until five cycles of freeze-thaw.
\end{abstract}

Conclusion: Therefore, the choice of organogelator governs the rheological, thermal, and drug diffusion properties of soy-gels intended for topical application.

Keywords: Gelation kinetics, non-Fickian diffusion, Organogel, Organogelator, Soybean oil, Span 40, Span 60, Viscosity.

(C) 2019 The Authors. Published by Innovare Academic Sciences Pvt Ltd. This is an open access article under the CC BY license (http://creativecommons. org/licenses/by/4. 0/) DOI: http://dx.doi.org/10.22159/ajpcr.2019.v12i6.33554

\section{INTRODUCTION}

Gels are suitable dosage forms for topical application due to their ease of manufacturing, excellent appearance, smoothness, desired consistency, convenience in handling, superior drug release profile, and finally affordability. They are composed of polymeric gelling component forming a closely entangled mesh of aggregates by immobilizing the liquid component [1]. Gels may be categorized as a hydrogel, emulgel, organogel or oleogel depending on the polarity of the liquid component [2]. Hydrogels are three-dimensional (3D), hydrophilic, polymeric networks capable of imbibing large amounts of water, or biological fluids [3]. However, they are highly susceptible to microbial contamination and also possess poor mechanical strength [4]. On the other hand, absorption of macromolecules from emulgels is low and bubble formation may occur during emulgel formulation [5].

Recently, attempts are being made to modify gel formulation by developing organogel which is solely a gelled homogeneous mixture of non-ionic surfactant with a non-polar organic solvent or vegetable oil at room temperature [6]. Organogel is a thermodynamically stable, viscoelastic system comprising of a gelator (any substance capable of forming gel) and a nonpolar phase (non-aqueous), with or without the presence of water molecules within the network formed by the gelator system [7]. Drug molecule may be incorporated either in the organic phase or sometimes in the aqueous component.

Gelators are organic molecules capable of gelling an organic phase at a definite temperature to form organogel [8]. Microscopy of the organogels showed that the 3D network of needle aggregates of gelator is responsible for immobilizing the apolar solvent. Non-ionic surfactants such as sorbitan esters or Spans are being currently employed for crippling the non-polar phase in pharmaceutical, food, and cosmetic industries [9]. Due to their low cost and stability over a wide range of temperature, sorbitan esters are quite often being used as an alternative to the phospholipids for various topical preparations [10]. Sorbitan esters are generally classified according to the presence of fatty acid chain in their chemical structure. Presence of long saturated hydrocarbon chains within the sorbitan ester molecules results in the formation of solid esters (e.g., sorbitan monopalmitate [SP or Span 40] and sorbitan monostearate [SS or Span 60]) whereas, the short hydrocarbon chains form liquid esters (e.g., sorbitan monolaurate or Span 20). SP and SS have been found to be biocompatible, odorless, and form opaque, thermoreversible semi-solid oleogels with apolar liquids such as vegetable oil (soybean oil, sunflower oil, mustard oil, groundnut oil, olive oil, etc.) and dichloromethane [7]. SS and SP based organogels have potential applications as delivery vehicles for drugs and antigens [11].

Satapathy et al. reported that the mustard oil and groundnut oil based Span 40 organogel was opaque, thermoreversible, pseudoplastic and thixotropic in nature, and has huge potential to be used as the controlled delivery vehicle. Thermal stability of groundnut oil based organogels was found to be more than the mustard oil based organogels [12]. Rushikesh et al. described the development of Span 60 based organogel using sunflower oil as an apolar phase [13]. Shah et al. (2013) reported that organogel was formed due to entanglement of olive oil molecules within the tubular structure of gelator molecules of Span 40 and Span 60. Differential scanning calorimetry (DSC) and gel disintegration studies suggested that the Span 60 based organogels were having higher thermal 
and physical strength as compared to the Span 40 based organogels [14]. The use of sorbitan esters for the development of organogels has thus opened up a new field of study.

Soybean oil-based organogel or soy-gel with Span 40 or Span 60 may be formulated as a topical base for drug incorporation due to soybean oil's stability to oxidation which is limited by its linolenic acid content [15]. In the present investigation, attempts were made to develop and compare sorbitan monopalmitate and sorbitan monostearate based soy-gels for topical applications.

Till date, no comparative analysis has been carried out on Span 40 and Span 60 based soy-gels which can enable choice of a suitable soybean-oil based topical base for controlled drug delivery for release of various active molecules. Since the two selected sorbitan esters are structurally different, they are expected to produce organogels having varying characteristics such as thermal properties, viscosity, and drug release behavior and may exhibit differences in stability profile.

\section{MATERIALS AND METHOD}

\section{Materials}

Food grade soybean oil (Emami Ltd., Kolkata, West Bengal, India) was purchased from the local market. Paracetamol IP (PCM) was received as a gift sample from the enlisted vendor. Span 40 (sorbitan monopalmitate, SP) and Span 60 (sorbitan monostearate, SS) were purchased from Loba Chemie Pvt. Ltd., Mumbai, Maharashtra, India. All other reagents were of analytical grade.

\section{Methods}

\section{Method of preparation of organogel}

Soybean oil was maintained at $60^{\circ} \mathrm{C}$ to which $2 \% \mathrm{w} / \mathrm{v}$ PCM was added slowly with constant stirring on mechanical stirrer (REMI) at $500 \mathrm{rpm}$. Organogelator (Span 40 or Span 60) was added to the oil and stirred for $1 \mathrm{~h}$ when a clear homogeneous solution was obtained. Subsequently, the mixture was allowed to cool down to $25^{\circ} \mathrm{C}$. If the mixture failed to flow when inverted vertically, it may be considered to form organogel. Span 40 (SP) and Span 60 (SS) based soy-gels were formulated using varying concentrations of each organogelator and composition is given in Table 1. The formulations were stored in glass vials at $25^{\circ} \mathrm{C}$ for further characterization after $72 \mathrm{~h}$. For each organogel, there exists a critical gelator concentration (CGC) which is regarded as the threshold gelator concentration at which the gelation is induced [16].

\section{Characterization of soy-gels}

\section{Fourier transform infrared (FTIR) study}

Compatibility study of blank as well as drug loaded organogel along with its individual components was carried out using FTIR Spectrometer (ALPHA-II, Bruker, Billerica, MA, USA) operated in attenuated total reflectance mode [17]. Samples were scanned in the range of 4000$500 / \mathrm{cm}$

\section{Organoleptic evaluation}

Organogels were observed visually for their color, appearance, texture, and opacity [18].

Table 1: Composition of organogels

\begin{tabular}{lllllllll}
\hline Ingredients (\%w/v) & SP1 & SP2 & SP3 & SP4 & SS1 & SS2 & SS3 & SS4 \\
\hline Soybean oil & 82 & 80 & 78 & 76 & 82 & 80 & 78 & 76 \\
PCM & 2 & 2 & 2 & 2 & 2 & 2 & 2 & 2 \\
Span 40 & 16 & 18 & 20 & 22 & - & - & - & - \\
Span 60 & - & - & - & - & 16 & 18 & 20 & 22 \\
\hline
\end{tabular}

Blank gels were named as SP* and SS* and used for characterization studies other than in vitro drug release study, *Indicates drug-free organogels.

SP: Sorbitan monopalmitate, SS: Sorbitan monostearate

\section{$p H$ determination}

The $\mathrm{pH}$ of the formulations was determined using a digital $\mathrm{pH}$ meter (Fisher Scientific-Accumet AE 150) [18].

\section{Extrudability}

A fixed weight of organogel was filled into an ointment tube and crimped. The extrudability $(\mathrm{cm} / \mathrm{s})$ of gel was determined by measuring the length of the gel ribbon extruded from the ointment tube by applying uniform pressure over a period of $10 \mathrm{~s}$ [19]. The following equation was used to determine extrudability.

$$
\text { Extrudability }=\text { Distance travelled by the gel }(\mathrm{cm}) / 10 \mathrm{~s}
$$

\section{Spreadability}

Spreadability of the formulations was determined by placing $0.1 \mathrm{~g}$ formulation between two glass slides of equal dimensions $(75 \mathrm{~mm}$ $\times 25 \mathrm{~mm} \times 1 \mathrm{~mm}$ ). Thereafter, known weights of $10 \mathrm{~g}, 20 \mathrm{~g}, 50 \mathrm{~g}$, or $100 \mathrm{~g}$ were loaded separately on the upper slide for $60 \mathrm{~s}$. The initial and final spreading diameters were marked before and after placing the weight [20]. Finally, the percentage spreadability may be calculated using the following equation (2).

$$
\% \text { Spreadability }=\left(\left[\mathrm{D}_{\mathrm{i}}-\mathrm{D}_{\mathrm{f}}\right] / \mathrm{D}_{\mathrm{i}}\right) \times 100
$$

Where, $\mathrm{D}_{\mathrm{i}}=$ initial spreading diameter, $\mathrm{D}_{\mathrm{f}}=$ final spreading diameter

\section{Viscosity}

The viscosity of the blank gels (SP* and SS*) was measured in Brookfield Digital Viscometer (Model LVDVI+, Brookfield Engineering Laboratories Inc, USA) with spindle no. 6 at $1 \mathrm{rpm}$ for $1 \mathrm{~min}$ at $25^{\circ} \mathrm{C}$ [21].

\section{Gelation kinetics study}

Gelation kinetics study of the blank soy-gels was carried out by measuring the intensity of turbidity against time elapsed. Initially, organogel was found to exist in a transparent sol state which became turbid with time due to the formation of a gel. Change from sol to gel state was reflected as an increase in nephelometric turbidity unit (NTU) as measured using a Nepheloturbidimeter (ELICO CL 52D Nephelometer). Soybean oil was taken as sample blank and the intensity of the turbidity of the organogel was measured at 10 $s$ intervals. The time at which turbidity attained a constant value is defined as gelation time. The result is represented graphically to observe the change in the gelation process with organogelator type and concentration.

Gompertz model was employed for modeling of gelation kinetics [22]. This non-linear model indicates a relationship between turbidity intensity (NTU), the concentration of gelator in $\% \mathrm{w} / \mathrm{v}(\rho)$ and time for gelation in $\mathrm{h}(\mathrm{x})$

$\alpha$ is defined as oil parameter and is related to gel flexibility whereas $\beta$ indicates a characteristic property related to organogelator or can be defined as organogelator parameter and is related to the thermal stability of gel.

$$
\log Y=\alpha+\beta \rho^{x}
$$

\section{Determination of gel-sol transition temperature}

The gel-sol transition temperature $\left(\mathrm{T}_{\mathrm{g}}\right)$ of the organogels was determined by the falling ball method [23]. Briefly, a metal ball of weight $250 \mathrm{mg}$ was placed gently on the surface of the soy-gel taken in a beaker. A thermometer was inserted in the gel, and the gel was heated from $25^{\circ} \mathrm{C}$ to $70^{\circ} \mathrm{C}$ at a rate of $1{ }^{\circ} \mathrm{C} / \mathrm{min}$. The temperature at which the ball started to move from the surface through the gel was recorded as the gel-sol transition temperature $\left(\mathrm{T}_{\mathrm{g}}\right)$. 


\section{Drug content determination}

A definite amount of drug-loaded soy-gel was mixed with phosphate buffer ( $\mathrm{pH}$ 5.8) to produce a uniform dispersion which was kept undisturbed for $48 \mathrm{~h}$ [24]. The dispersion was filtered through Whatman filter paper No. 1. An aliquot of the filtrate was suitably diluted and absorbance measured spectrophotometrically at $249 \mathrm{~nm}$ (Shimadzu UV-VIS 1800 spectrophotometer) [25]. The drug content of formulations was determined from the calibration curve of the drug in the said buffer.

\section{In vitro drug release study}

In vitro drug release from organogels was performed through a dialysis membrane (HIMEDIA ${ }^{\circledR}$ LA 330-5MT) in modified Franz diffusion cell [26]. Accurately weighed drug-loaded sample containing drug equivalent to $4 \mathrm{mg}$ was placed in the donor compartment and the receptor chamber containing phosphate buffer $(\mathrm{pH}$ 5.8) was maintained at $32^{\circ} \mathrm{C} \pm 0.5^{\circ} \mathrm{C}$. An aliquot of $1 \mathrm{ml}$ was withdrawn every hour, replenished with fresh buffer and study was continued for $6 \mathrm{~h}$. The aliquot was analyzed spectrophotometrically at $249 \mathrm{~nm}$ (Shimadzu UV-VIS 1800 spectrophotometer) [25].

Drug release data were subjected to mathematical modeling using zeroorder, first-order, Higuchi, and Korsmeyer-Peppas model [27].

\section{Hemocompatibility study [28]}

Accurately weighed the amount of blank organogel was placed in dialysis tube filled with $50 \mathrm{ml}$ normal saline $(0.9 \% \mathrm{w} / \mathrm{v} \mathrm{NaCl}$ solution $)$ and continuously stirred in a magnetic stirrer for $1 \mathrm{~h}$ at $37^{\circ} \mathrm{C}$ to enable leaching of the soy-gel components. Leachant $(0.5 \mathrm{ml})$ was withdrawn, diluted to $10 \mathrm{ml}$ with normal saline and $0.5 \mathrm{ml}$ diluted goat blood $(4 \mathrm{ml}$ of goat blood diluted with $5 \mathrm{ml}$ of normal saline) and incubated at $37^{\circ} \mathrm{C}$ for $1 \mathrm{~h}$. It was then centrifuged at $3000 \mathrm{rpm}$ for $10 \mathrm{~min}$. The supernatant was measured spectrophotometrically at $542 \mathrm{~nm}$. The positive control was prepared by taking $0.1(\mathrm{~N}) \mathrm{HCl}$ solution in lieu of leachant. In the negative control, normal saline was used instead of leachant. Normal saline was taken as the corresponding blank and percent hemolysis may be calculated as follows:

$$
\% \text { Hemolysis }=\left(\mathrm{OD}_{\text {test }}-\mathrm{OD} \text { negative }\right) /\left(\mathrm{OD}_{\text {positive }}-\mathrm{OD} \mathrm{n}_{\text {negative }}\right) \times 100
$$

\section{Table 2: Organoleptic characterization of Span based soy-gels}

\begin{tabular}{|c|c|c|c|c|}
\hline Formulation & Color & Odor & Appearance & Opacity \\
\hline SP1* & - & - & - & - \\
\hline SP2 & Yellowish white & Odorless & Smooth-oily & Slightly opaque \\
\hline SP3 & Yellowish white & Odorless & Smooth-oily & Slightly opaque \\
\hline SP4 & Yellowish white & Odorless & Smooth-oily & Slightly opaque \\
\hline SS1 & Creamy white & Odorless & Smooth-oily & Opaque \\
\hline SS2 & Creamy white & Odorless & Smooth-oily & Opaque \\
\hline SS3 & Creamy white & Odorless & Smooth-oily & Opaque \\
\hline SS4 & Creamy white & Odorless & Smooth-oily & Opaque \\
\hline
\end{tabular}

*Indicates no gel formation. SP: Sorbitan monopalmitate, SS: Sorbitan monostearate
Where,

$\mathrm{OD}_{\text {test }}=$ Absorbance of the test sample

$\mathrm{OD}_{\text {positive }}=$ Absorbance of the positive control

$\mathrm{OD}_{\text {negative }}^{\text {positive }}=$ Absorbance of the negative control

\section{Accelerated thermal stability study}

The stability analysis of the pharmaceutical products may be carried out either by a thermocycling process or by incubating the samples at a specific environment for a longer period of time [29].

Accelerated thermal stability studies of Span 40 and Span 60 based soygels were carried out by thermocycling method [14]. Freshly prepared organogels were subjected to 5 consecutive freeze-thaw cycles. During each cycle, organogels were heated to $65^{\circ} \mathrm{C}$ and then immediately kept overnight at $4^{\circ} \mathrm{C}$.

\section{Statistical analysis}

Data have been obtained from each experiment in triplicate $(n=3)$ and were subjected to statistical analysis using one-way analysis of variance [30]. Results are quoted as significant where $\mathrm{p}<0.05$.

\section{RESULTS}

\section{Organogel formation}

The CGC for Span 60 was found to be 16\% w/v (SS1) and 18\%w/v for Span 40 with soybean oil (SP2).

\section{Organoleptic evaluation}

Span 40 and Span 60 based soy-gels were found to be closely identical in their organoleptic properties except color and degree of opacity (Table 2). Increase in consistency on increasing concentration of the organogelator was visible in both Span 40 and Span 60 based soy-gels. $\mathrm{pH}$ of the formulations was found to be between 5.5 and 5.8 at $25^{\circ} \mathrm{C}$ which is compatible with skin.

\section{FTIR study}

FTIR study of blank as well as drug-loaded organogels revealed the characteristic peaks of individual gel components and drug. The FTIR spectral analysis of Span 40 and Span 60 showed a $0-\mathrm{H}$ stretching peak at 3300/cm whereas Span 40 and Span 60 based organogels did not show any $\mathrm{O}-\mathrm{H}$ stretching vibration at $3300 / \mathrm{cm}$ (Fig. 1).

\section{Extrudability and spreadability study}

Extrudability of the Span 40 and Span 60 based organogels was found to be satisfactory. The percent spreadability of Span 40 and Span 60 based organogels is shown in Table 3 .

\section{Viscosity}

Different organogel formulations showed concentration-dependent increase in viscosity (Table 4). Viscosity of Span 60 based soygels was found to be 10 times higher than that of Span 40 based formulations.

Table 3: Extrudability, spreadability, and hemocompatibility studies on Span based soy-gels

\begin{tabular}{|c|c|c|c|c|c|c|}
\hline \multirow[t]{2}{*}{ Formulation } & \multirow[t]{2}{*}{ Extrudability* $(\mathrm{cm} / \mathrm{s})$} & \multicolumn{4}{|c|}{ Percentage spreadability on application of } & \multirow[t]{2}{*}{ Hemocompatibility } \\
\hline & & $10 \mathrm{~g}$ & $20 \mathrm{~g}$ & $50 \mathrm{~g}$ & $100 \mathrm{~g}$ & \\
\hline SP2 & $0.7 \pm 0.32$ & 15.72 & 27.12 & 65.52 & 85.62 & Pass \\
\hline SP4 & $0.6 \pm 0.41$ & 13.56 & 22.09 & 45.98 & 53.89 & Pass \\
\hline SS1 & $0.76 \pm 0.31$ & 36.92 & 45.92 & 77.16 & 97.60 & Pass \\
\hline SS2 & $0.75 \pm 0.24$ & 42.73 & 62.85 & 75.89 & 88.18 & Pass \\
\hline SS3 & $0.78 \pm 0.35$ & 37.49 & 45.26 & 74.85 & 92.76 & Pass \\
\hline SS4 & $0.76 \pm 0.16$ & 33.33 & 46.15 & 57.89 & 97.43 & Pass \\
\hline
\end{tabular}

*Data presented as mean \pm SE of mean from $n=3, p<0.05$ indicating statistically significant differences. SE: Standard deviation, SP: Sorbitan monopalmitate, SS: Sorbitan monostearate 


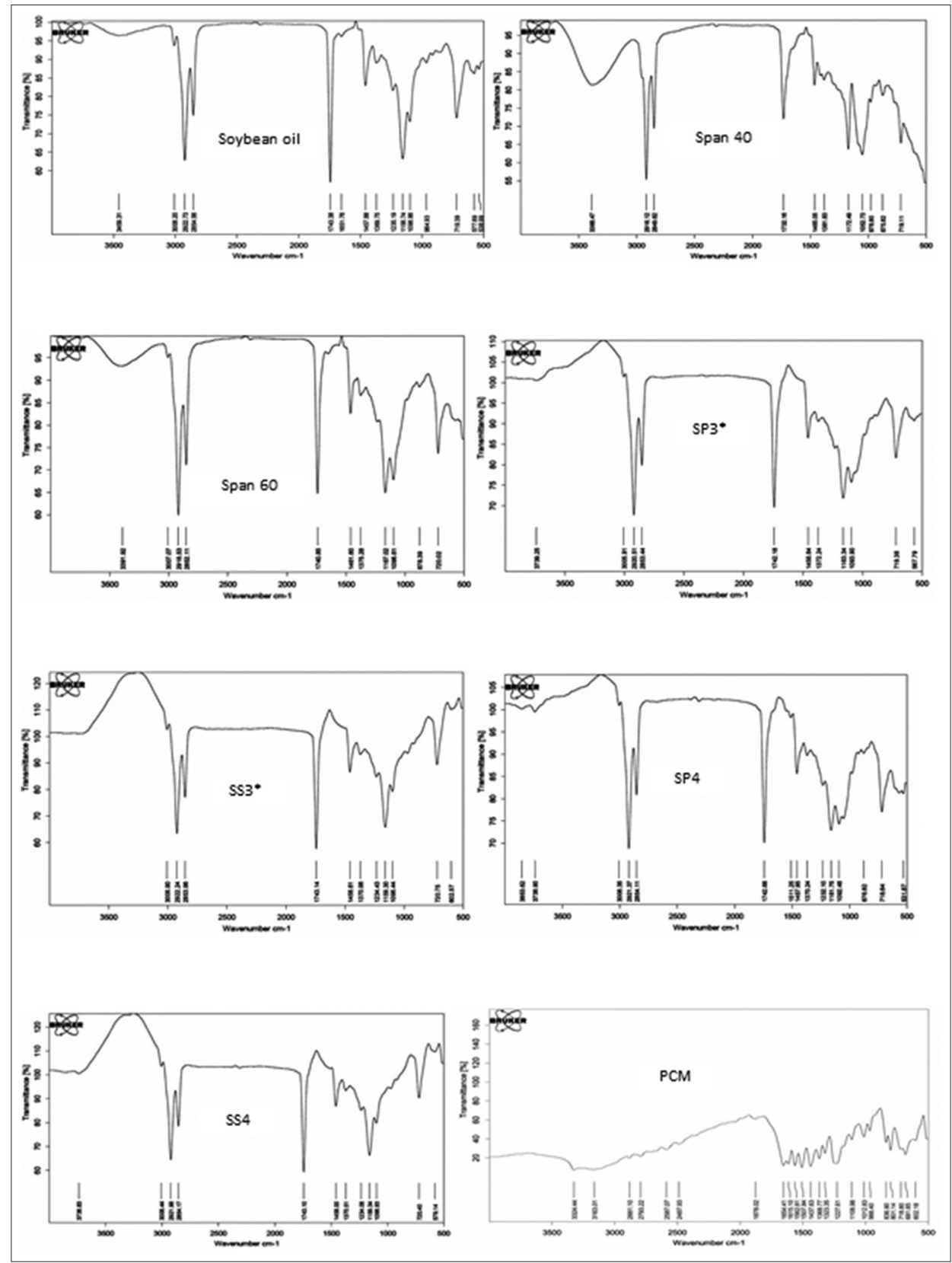

Fig. 1: Fourier transform infrared spectroscopic analysis of soy-gel components, blank gels (SP* and SS*) and drug-loaded soy-gels

Table 4: Viscosity study and thermal analysis of Span based soy-gels

\begin{tabular}{|c|c|c|c|}
\hline Formulation & $\begin{array}{l}\text { Viscosity at } \\
25^{\circ} \mathrm{C} \text { (cp) }\end{array}$ & $\begin{array}{l}\text { Gel-sol transition } \\
\text { temperature }(\mathrm{Tg})\left({ }^{\circ} \mathrm{C}\right)\end{array}$ & $\begin{array}{l}\text { Gelation } \\
\text { time (min) }\end{array}$ \\
\hline SP2 & $3.9 \times 10^{4}$ & 42 & 17 \\
\hline SP3 & $4.43 \times 10^{4}$ & 46 & 8 \\
\hline SP4 & $5.02 \times 10^{5}$ & 48 & 7 \\
\hline SS1 & $2 \times 10^{5}$ & 56 & 6 \\
\hline SS2 & $2.4 \times 10^{5}$ & 57 & 4 \\
\hline SS3 & $3 \times 10^{5}$ & 59 & 3 \\
\hline SS4 & ND* & 63 & 3 \\
\hline
\end{tabular}

*Viscosity of SS 4 (22\% w/v Span 60$)$ could not be determined with spindle 6 and at $1 \mathrm{rpm}$, hence not given. ND: Not determined, SP: Sorbitan monopalmitate, SS: Sorbitan monostearate

\section{Gelation kinetics study}

The parameters related to soy-gel flexibility and thermal stability, $\alpha$ and $\beta$ are reported in Table 5 .
Table 5: Modeling of gelation kinetics of span based soy-gels

\begin{tabular}{lll}
\hline Formulation & \multicolumn{2}{l}{ Gompertz model } \\
\cline { 2 - 3 } & $\boldsymbol{\alpha}$ & $\boldsymbol{\beta}$ \\
\hline SP2 & 2.072 & 0.5791 \\
SP3 & 2.062 & 0.8454 \\
SP4 & 1.0197 & 1.8156 \\
SS1 & 0.8753 & 0.1313 \\
SS2 & 0.7296 & 0.1565 \\
SS3 & 0.6223 & 0.1682 \\
SS4 & 0.3410 & 0.2903 \\
\hline
\end{tabular}

SP: Sorbitan monopalmitate, SS: Sorbitan monostearate

Determination of gel-sol transition temperature

Gel-sol transition temperature $\left(\mathrm{T}_{\mathrm{g}}\right)$ of Span 40 based soy-gels was found to be in the range of $42^{\circ} \mathrm{C}-48^{\circ} \mathrm{C}$ whereas it was in the range of $56^{\circ} \mathrm{C}-63^{\circ} \mathrm{C}$ in case of Span 60 based formulations (Table 4 ). 
Drug content study

Drug content of SP-based soy-gels was found to be in the range 95-98\% and it was lower (90-93\%) in SS based soy-gels.

\section{In vitro drug release study}

In vitro, drug release study revealed $50.81 \%$ drug release from SP2 in $6 \mathrm{~h}$ and $47.34 \%$ drug released from Span 60 based soy-gel at its CGC (SS1; 16\% w/v) (Fig. 2). Increase in organogelator concentration reduced drug release. Soy-gels with a higher concentration of Span 40 (SP3 and SP4) followed Korsmeyer-Peppas kinetics with non-Fickian diffusion. At CGC, Span 40-based soy-gel (SP2) released the drug by Fickian diffusion. Span 60 based soy-gels manifested remarkable change in release kinetics as three formulations $(16-20 \% \mathrm{w} / \mathrm{v}$ Span 60) (SS1, SS2, and SS3) followed zero-order kinetics with non-Fickian diffusion except SS4 which followed Korsmeyer-Peppas model (Table 6).

\section{Hemocompatibility study}

The percentage hemolysis of all the formulations was found to be $<5 \%$ in presence of organogel leachant (Table 3).

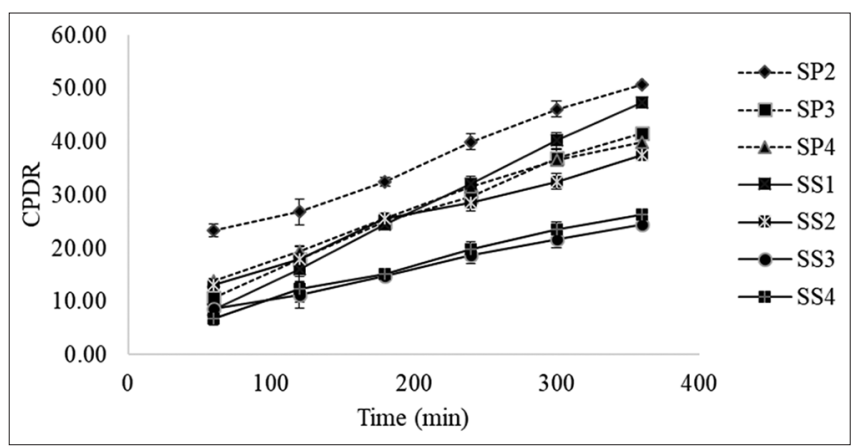

Fig. 2: Drug release profile of Span based soy-gels. CPDR represents cumulative percentage drug released from soy-gels. Data presented as mean \pm standard error of mean from $n=3$. $\mathrm{p}<0.05$ indicating statistically significant differences

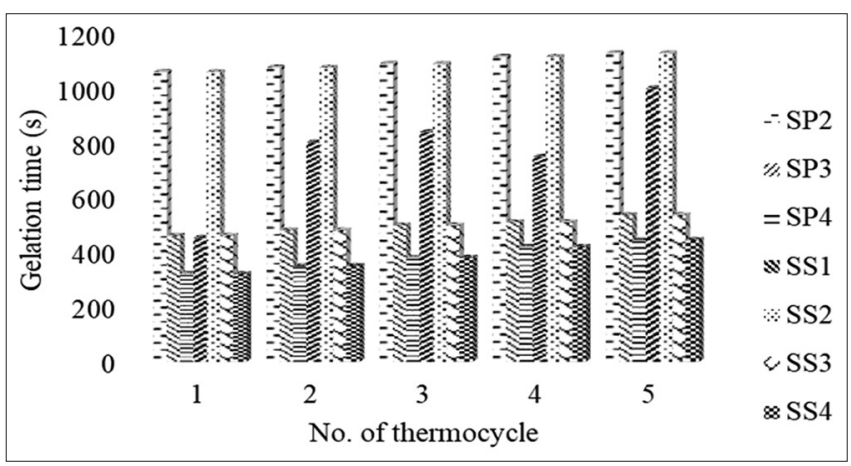

Fig. 3: Accelerated thermal stability study of soy-gels subjected to five freeze-thaw cycles
Accelerated thermal stability study

Change in gelation time after each thermocycler of freeze-thaw for Span 40 and Span 60 based soy-gel has been graphically represented to determine gel stability after subjecting to specified temperature fluctuations (Fig. 3).

\section{DISCUSSION}

Formation of soy-gel occurred owing to lowering of the solubility parameter of Span 40 and Span 60 and precipitation of the gelator molecules as the temperature was reduced. This may be due to the interaction between the hydrophobic components of the gelator fibers thereby forming a 3D network structure of gelator, and immobilized soybean oil with drug molecules entrapped within solid fiber organogels [31]. The difference in CGC was noted for soybean oil with a change in Span indicating difference in the ability to induce gelation with change in structure of organogelator [32]. FTIR study indicated compatibility among organogel components and also the existence of PCM in native state within drug-loaded gel. Absence of $\mathrm{O}-\mathrm{H}$ peak in organogel indicates recession of the $\mathrm{O}-\mathrm{H}$ stretching within the gelator molecules [33].

Extrudability is one of the important mechanical characteristics of a gel which confirms the structural behavior on application of fixed pressure into a specific area. Spreadability is behavior of a gel that ensures mechanical strength and total integrity upon application. Soy-gels formulated with Span 40 and Span 60 are expected to spread uniformly over the affected area of skin on being extruded without loss of structural integrity. The formulations may be regarded as hemocompatible as percentage hemolysis was $<5 \%$ [34].

Viscosity is an important property of gel-like formulations [35]. Addition of Span 60 increased the viscosity of soy-gels probably due to its better ability to form gels in comparison to Span 40 .

As the temperature of the organogels was increased, there was a corresponding increase in surface free energy with subsequent increase in mobility of the gelator molecules constituting the 3D-self assembled structure of the formulations. With further increase in temperature the interaction between self-assembled structure was totally disrupted leading to the breakdown of networked structure, thereby causing the gelled system to acquire sol state and flow freely [36]. It is to be noted that gel-sol transition temperature of Span 40-based organogels was considerably lower relative to corresponding Span 60-based formulations. Moreover, gelation times were lower with Span 60 based soy-gels. In both the cases, gel-sol transition temperature increased and gelation time decreased with increase in concentration of organogelator (Table 4). The differences in organogel behavior may be attributed to better networking ability of Span 60 compared to Span 40. Critical comparison of Span 40-and Span 60-based soy-gels was done on the basis of gelation kinetics study through the determination of $\alpha$ and $\beta$ parameters related to gel flexibility and thermal stability of the soy-gels, respectively. As the concentration of gelator was increased in both Span 40 and Span 60 based soy-gels, $\alpha$ value (flexibility) decreased indicating formation of comparatively rigid gels with higher viscosity (SP4 and SS4) and

Table 6: Modeling of drug release kinetics from Span based soy-gels

\begin{tabular}{|c|c|c|c|c|}
\hline \multirow[t]{2}{*}{ Formulation } & \multirow{2}{*}{$\begin{array}{l}\text { Zero-order model } \\
R^{2}\end{array}$} & \multicolumn{2}{|c|}{ Korsmeyer-Peppas model } & \multirow[t]{2}{*}{ Best fit model } \\
\hline & & $R^{2}$ & $\mathbf{n}$ & \\
\hline SP2 & 0.9909 & 0.9438 & 0.45 & Zero-order with Fickian diffusion \\
\hline SP3 & 0.995 & 0.9988 & 0.76 & Korsmeyer-peppas with non-Fickian diffusion \\
\hline SP4 & 0.985 & 0.9934 & 0.60 & Korsmeyer-peppas with non-Fickian diffusion \\
\hline SS1 & 0.999 & 0.745 & 0.77 & Zero-order with non-Fickian diffusion \\
\hline SS2 & 0.999 & 0.755 & 0.57 & Zero-order with non-Fickian diffusion \\
\hline SS3 & 0.996 & 0.960 & 0.56 & Zero-order with non-Fickian diffusion \\
\hline SS4 & 0.979 & 0.9941 & 0.7261 & Korsmeyer-peppas with non-Fickian diffusion \\
\hline
\end{tabular}

SP: Sorbitan monopalmitate, SS: Sorbitan monostearate 
better thermal stability as manifested in increasing $\beta$-value and higher $\mathrm{T}_{g}$ (Table 4). Soy-gels containing Span 40 as organogelator were found to possess higher flexibility $(\alpha)$ compared to corresponding Span 60 based soy-gels. Although Span 40 gels are having a higher $\beta$ value indicating higher thermal stability, the $\mathrm{T}$ values of Span 60 gels were found to be higher with corresponding lower $\beta$-values. This can be explained by the ability of Span 60 to impart higher thermal stability to gels at comparatively lower $\beta$ value which indicates firmness of the structure attributed by Span 60 [14,31]. A similar report of better thermal strength of Span 60 based olive oil organogels is already existing in literature [14]. Since, Span 60 was found to show lower CGC $(16 \% \mathrm{w} / \mathrm{v})$ compared to Span $40(18 \% \mathrm{w} / \mathrm{v})$ for soy-gel and imparts better thermal stability, Span 60 may be assumed to have modified microarchitecture of soy-gels. The Span 40 based formulations with higher $\alpha$ value (SP2 and SP3) and lower viscosity are expected to exhibit improved drug release.

Drug release data from Span 40 and Span 60 based soy-gels revealed major differences. All the formulations released approximately 40-50\% drug (PCM) in $6 \mathrm{~h}$ except SS2, SS3, and SS4. SS1 (16\%w/v Span 60) demonstrated better release than SP3 and SP4. It is to be noted that enhanced drug release was found from Span 40 based soy-gels as expected from gelation kinetics study and viscosity data. Improved drug release is thus attributed to the flexible structure of Span 40 gels and probably amorphous nature of the gels [37]. Poor drug release from soy-gels necessitates the development of strategies to improve organogel formulation.

Kinetic modeling of drug release data revealed non-Fickian drug diffusion from gels with increasing concentration of Span 40 (with $20 \% \mathrm{w} / \mathrm{v}$ and $22 \% \mathrm{w} / \mathrm{v}$ Span 40 ) which exhibited KorsmeyerPeppas model. However, zero-order drug release or nearly constant concentration-independent release was observed in SP2 and all Span 60 formulations except SS4. Fickian diffusion was detected only in soy-gels with the least concentration of Span 40 . Ideally, the lowest concentration of Span 40 gels $(18 \% \mathrm{w} / \mathrm{v})$ and Span 60 gels $(16-20 \% \mathrm{w} / \mathrm{v})$ can be selected as topical bases for controlled drug release [38].

The formulations were found to be stable after five thermocycles with no visible signs of instability. However, the gelation time of both SP and SS based soy-gels was increased gradually. It may be said that there was a change in the network structure of the gels due to consecutive heating and cooling.

\section{CONCLUSION}

From the above studies, it can thus be concluded that type and concentration of organogelator has a profound influence on the viscosity, flexibility, thermal stability, and drug release behavior of soybean oil-based soy-gel intended for topical application to achieve controlled release.

\section{AUTHORS' CONTRIBUTIONS}

The present research work is a part of M Pharm thesis work of first two authors under the guidance of last 2 authors and all have equally contributed in writing and editing the manuscript at various stages.

\section{CONFLICTS OF INTEREST}

All authors have none to declare.

\section{REFERENCES}

1. Murdan S. Organogels in drug delivery. Expert Opin Drug Deliv 2005;2:489-505.

2. Kaur LP, Gulen TK. Topical gel: A recent approach for novel drug delivery. Asian J Biomed Pharm Sci 2013;3:1-5.

3. Peppas NA, Bures P, Leobandung W, Ichikawa H. Hydrogels in pharmaceutical formulations. Eur J Pharm Biopharm 2000;50:27-46.

4. Hoffman AS. Hydrogels for biomedical applications. Adv Drug Del Rev 2012;64:18-23.
5. Sah SK, Badola A, Nayak BK. Emulgel: Magnifying the application of topical drug delivery. Indian J Pharm Bio Res 2017;5:25-33.

6. Jatav MP, Mandlekar R, Ramteke S. Formulation and evaluation of lecithin organogel for treatment of arthritis. Int J Adv Sci Res 2017; 1:300-7.

7. Purohit B, Gupta N, Jain S. Formulation and evaluation of diclofenac sodium organogel. Res J Pharm Tech 2013;6:375-8.

8. Garg T, Bilandi A, Kapoor B, Kumar S, Joshi R. Organogels: Advanced and novel drug delivery system. Int Res J Pharm 2011;2:15-21.

9. Marquez AL, Palazolo GG, Wagner JR. Water in oil (w/o) and double (w/o/w) emulsions prepared with Spans: Microstructure, stability, and rheology. Coll Polym Sci 2007;285:1119-28.

10. Asthana GS, Sharma PK, Asthana A. In vitro and in vivo evaluation of niosomal formulation for controlled delivery of clarithromycin. Scientifica (Cairo) 2016;2016:6492953.

11. Dassanayake LSK, Kodali DR, Ueno S. Formation of oleogels based on edible lipid materials. Curr Opin Colloid Interface Sci 2011;16:432-9.

12. Satapathy D, Sagiri SS, Pal K, Pramanik K. Development of mustard oil-and groundnut oil-based Span 40 organogels as matrices for controlled drug delivery. Des Monomers Polym 2014;16:545-56.

13. Rushikesh $\mathrm{P}$, Abraham S, Bharath S, Madhavan V. Sorbitan monostearate based organogels for topical delivery of clotrimazole. Int J Pharm Chem Res 2013;2:1246-52.

14. Shah DK, Sagiri SS, Behera B, Pal K, Pramanik K. Development of olive oil based organogels using sorbitan monopalmitate and sorbitan monostearate: A comparative study. J Appl Polym Sci 2013;129:793805 .

15. Hammond EG, Johnson LA, Caiping S, Wang T, White PJ. Soybean oil. In: Fereidoon S, editor. Bailey's Industrial Oil and Fat Products. $6^{\text {th }}$ ed. USA: John Wiley and Sons, Inc.; 2005. p. 584-91.

16. Singh VK, Pramanik K, Ray SS, Pal K. Development and characterization of sorbitan monostearate and sesame oil-based organogels for topical delivery of antimicrobials. AAPS Pharm Sci Tech 2015;16:293-305.

17. Sagiri SS, Behera B, Sudheep T, Pal K. Effect of composition on the properties of Tween-80-Span-80-based organogels. Des Monomers Polym 2012;15:253-73.

18. Fayez SM, Gad S, Khafagy SA, Jaleel GA, Ghorab MM, El-Nahhas SA. Formulation and evaluation of etodolac lecithin organogel transdermal delivery systems. Int J Pharm Pharm Sci 2015;7:325-34.

19. Sah SK, Badola A, Mukhopadhyay S. Development and evaluation of tioconazole loaded emulgel. Int J Appl Pharm 2017;9:83-90.

20. Reza MI, Goel D, Gupta RK, Warsi MH. Formulation of ketoconazole loaded nano dispersive gel using swollen micelles technique and its in vitro characterization. Int J Pharm Pharm Sci 2018;10:162-6.

21. Penzes T, Csoka I, Eros I. Rheological analysis of the structural properties effecting the percutaneous absorption and stability in pharmaceutical organogels. Rheol Acta 2014;43:457-63.

22. Shukla AK, Yadav SK, Tiwari V. Linear models for S-shaped growth curves. J Stat Appl Proc 2015;4:113-7.

23. Takahashi A, Sakai M, Kato T. Melting temperature of thermally reversible gel. VI. Effect of branching on the sol-gel transition of polyethylene gels. Poly J 1980;12:335-41.

24. Balata GF, Shamard HA El-M. Formulation of chlorpheniramine maleate in Span 60/Tween 20 based organogels for transdermal delivery. J Innov Pharm Bio Sci 2017;4:49-57.

25. Government of India. Indian Pharmacopoeia: Government of India, Ministry of Health and Family Welfare Department. $7^{\text {th }}$ ed. Government of India; 2014. p. 559-621.

26. Sagiri SS, Kasiviswanathan U, Shaw GS, Singh M, Anis A, Pal K. Effect of sorbitan monostearate concentration on the thermal, mechanical and drug release properties of oleogels. Korean J Chem Eng 2016;33:1720-7.

27. Dash S, Murthy PN, Nath L, Chowdhury P. Kinetic modeling on drug release from controlled drug delivery systems. Acta Pol Pharm 2010; 67:217-23

28. Pal K, Banthia AK, Majumdar DK. Biomedical evaluation of polyvinyl alcohol-gelatin esterified hydrogel for wound dressing. J Mater Sci Mater Med 2007;18:1889-94.

29. Chen Z, Li F, Yang H, Yi T, Huang C. A thermostable and long-termstable ionic-liquid-based gel electrolyte for efficient dye-sensitized solar cells. Chemphyschem 2007;8:1293-7.

30. Noorjahan CM, Green synthesis, characterization, and antibacterial activity of zinc oxide nanoparticle. Asian J Pharm Clin Res 2019;12:106-10.

31. Vintiloiu A, Leroux JC. Organogels and their use in drug delivery a review. J Contr Rel 2008;125:179-92.

32. Mercurio DJ, Spontak RJ. Morphological characteristics of 1, 3: 2, 
4-dibenzylidene sorbitol/poly (propylene glycol) organogels. J Phys Chem B 2001;105:2091-8.

33. Tata M, John VT, Waguespack YY, McPherson GL. A spontaneous phase transition from reverse miceiles to organogels due to surfactant interactions with specific benzenediols. J Mol Liq 1997;72:121-35.

34. Dutta P, Dey J, Perumal V, Mandal M. Amino acid based amphiphilic copolymer micelles as carriers of non-steroidal anti-inflammatory drugs: Solubilization, in vitro release and biological evaluation. Int J Pharm 2011;407:207-16.

35. Shchipunov YA, Mezzasalma SA, Koper GJM, Hoffmann H. Lecithin organogelwithnew rheologicaland scaling behavior.JPhys ChemB2001; 105:10484-8.

36. Jibry N, Heenan RK, Murdan S. Amphiphilogels for drug delivery: Formulation and characterization. Pharm Res 2004;21:1852-61.

37. Upadhyay KK, Tiwari C, Khopade AJ, Bohidar HB, Jain SK. Sorbitan ester organogels for transdermal delivery of sumatriptan. Drug Dev Ind Pharm 2007;33:617-25.

38. Papadopoulou V, Kosmidis K, Vlachou M, Macheras P. On the use of the Weibull function for the discernment of drug release mechanisms. Int J Pharm 2006;309:44-50. 